\title{
Generating Certain Class of Real Sequences Using Gamma Function, Arithmetic and Geometric Progressions
}

\author{
Vishwa Nath Maurya ${ }^{1, *}$, Ram Bilas Misra ${ }^{2}$, Chandra K. Jaggi ${ }^{3}$, Avadhesh Kumar Maurya ${ }^{4}$, \\ Rajnesh Krishnan Mudaliar ${ }^{5}$ \\ ${ }^{1}$ Professor \& Head, Department of Pure \& Applied Mathematics and Statistics, School of Science \& Technology, The University of \\ Fiji, Fiji Islands \\ ${ }^{2}$ Professor of Applied Mathematics, State University of New York, Korea Ex Vice-Chancellor, Dr. Ram Manohar Lohia Avadh \\ University, Faizabad, UP, India \\ ${ }^{3}$ Professor \& Head, Department of Operations Research, University of Delhi, New Delhi, India \\ ${ }^{4}$ Assistant Professor \& Head, Department of Electronics \& Communication Engineering, Lucknow Institute of Technology, Gautam \\ Buddha Technical University, Lucknow, U.P., India \\ ${ }^{5}$ Lecturer, Department of Mathematics \& Statistics, Fiji National University, Lautoka, Fiji Islands \\ *Corresponding author: prof.drvnmaurya@gmail.com
}

Received November 01, 2014; Revised November 10, 2014; Accepted November 21, 2014

\begin{abstract}
Present paper envisages a novel approach to explore some real sequences by using the gamma function, arithmetic progression (AP) and geometric progression (GP). Particularly, applications of properties of both the arithmetic progression (AP) and geometric progression (GP) are focused to find out some real sequences which can be significantly useful in emerging fields of engineering science and technology. Real sequences play vital role in testing of convergence of infinite power series in real analysis and engineering mathematics. In this paper, several propositions pertaining to real sequences are explored and examined by way of presenting proofs. In addition to this, various numerical examples are also illustrated in order to emphasis the application aspect of real sequences explored herein. Finally, some significant conclusions are drawn for future scope and further findings with different versions of real sequences.
\end{abstract}

Keywords: real sequence, $n^{\text {th }}$ term, complex number, gamma function, arithmetic progression, geometric progression

Cite This Article: Vishwa Nath Maurya, Ram Bilas Misra, Chandra K. Jaggi, Avadhesh Kumar Maurya, and Rajnesh Krishnan Mudaliar, "Generating Certain Class of Real Sequences Using Gamma Function, Arithmetic and Geometric Progressions.” American Journal of Applied Mathematics and Statistics, vol. 2, no. 6A (2014): 1-5. doi: 10.12691/ajams-2-6A-1.

\section{Introduction}

Sequences have an ancient history dating back at least as far as Archimedes who used sequences and series in his "Method of Exhaustion" to compute better values of $1 / 4$ and areas of geometry. A real sequence is a sequence where the subsequent segments are exact transpositions of the first segment. A tonal sequence is a sequence where the subsequent segments are diatonic transpositions of the first segments. A modified sequence is a sequence where the subsequent segments are decorated or embellished so as to not destroy the character of the original segment. A false sequence is a literal repetition of the beginning of a figure and stating the rest in sequence, we refer Benward and Saker [2]. A modulating sequence is a sequence that leads from one tonal center to the next, with each segment technically being in a different key in some sequences.
A sequence can be described according to its direction (ascending or descending in pitch) and its adherence to the diatonic scale that is, the sequence is diatonic if the pitches remain within the scale, or chromatic (or nondiatonic) if pitches outside of the diatonic scale are used and especially if all pitches are shifted by exactly the same interval (i.e., they are transposed). The non-diatonic sequence tends to modulate to a new tonality or to cause temporarily tonicisation.

At least two instances of a sequential pattern including the original statement are required to identify a sequence, and the pattern should be based on several melody notes or at least two successive harmonies (chords). Although stereotypically associated with Baroque music, and especially the music of Antonio Vivaldi, this device is widespread throughout Western music history.

The device of sequence epitomizes both the goaldirected and the hierarchical nature of common-practice tonality. It is particularly prevalent in passages involving extension or elaboration; indeed, because of its inherently 
directed nature, it was (and still is) often pulled from the shelf by the less imaginative tonal composer as the stock response to a need for transitional or developmental activity. Whether dull or masterly, however, the emphasis is on the underlying process rather than the material itself.

Basically, sequences are countably many numbers arranged in an ordered set that may or may not exhibit certain patterns.

A sequence is usually defined as a function whose domain is a countable totally ordered set, although in many disciplines the domain is restricted, such as to the natural numbers. In real analysis a sequence is a function from a subset of the natural numbers to the real numbers. In other words, a sequence is a map $f(n): N \rightarrow R$. We might identify $a_{n}=f(n)$ for all $n$ or just write $a_{n}: N \rightarrow R$.

Mathematical sequences can be used to model real life applications. Sequences and patterns arise naturally in many real life situations. Here is a stunning example to introduce the topic. Suppose you want to construct a movie theatre in your town. The number of seats in each row can be modelled by the formula $C(n)=16+4 n$, when $\mathrm{n}$ refers to the $n^{\text {th }}$ row, and you need 50 rows of seats.

(i). Write the sequence for the number of seats for the first 5 rows

(ii). How many seats will be in the last row?

(iii). What will be the total number of seats in the theatre?

Real sequences have vital role to generate formula and series in real analysis. Given an infinite sequence of numbers $\left\{a_{n}\right\}$, a series is informally the result of adding all those terms together: $a_{1}+a_{2}+a_{3}+\ldots$. These can be written more compactly using the summation symbol $\sum$. An example is the famous series from Zeno's dichotomy and its mathematical representation:

$$
\sum_{n=1}^{\infty} \frac{1}{2^{n}}=\frac{1}{2}+\frac{1}{4}+\frac{1}{8}+\cdots
$$

The terms of the series are often produced according to a certain rule, such as by a formula, or by an algorithm. For some well-known examples, we mention here the exponential series, Taylor's series, Maclaurin's series, and Fourier's series. As for as the application of sequences is concerned, we refer some other applications in music and communication technology e.g. Abolbashari and Aghaeinia [1], Benward and Saker [2], Caplin [3], GuuChang [5], Jue et al. [8], Kelly [9] and Sarnecki, Mark [12].

On the subject of sequence, numbers of textbooks are worth mentioning; for example, Dawikins and Paul [4], Hazewinkel and Michiel [6], Howard et al. [7] and Nico M. Temme [11].

Obtaining the terms of a real sequence when the $n^{\text {th }}$ term is known, is a mathematical science whereas finding the $n^{\text {th }}$ term of a sequence given some terms of a sequence is both a mathematical innovation and creative process. Over many years, the issue of finding the $n^{\text {th }}$ term of real sequences given some terms of the sequence is quite a herculean task and is in no way easy to come by. It is based on this premise that we present this work which is not in any way all-encompassing, aimed at addressing this problem to a certain degree by providing $n^{\text {th }}$ term of some classes of real sequences often encountered and consequently find other terms of such sequences.

\section{Preliminary Ideas and Definitions}

In order to serve our present objective to explore some real sequences, firstly we define few related terminologies. We begin with definition of terms:

Definition 2.1 A real sequence $\left\{a_{n}\right\}_{n \in \mathbb{N}}$ is a function $f: D \subseteq \mathbb{N} \rightarrow \mathbb{R}$ such that $f(n)=a_{n}, \forall n \in D$.

Definition 2.2 Let $f: \mathbb{N} \rightarrow \mathbb{R}$ be a real sequence, then $f(n)=a_{n}, \forall n \in \mathbb{N}$, called the $n^{\text {th }}$ term of the sequence is a formula in terms of $n$ which gives the value of the function at any $n \in \mathbb{N}$.

Definition 2.3 The Gamma function $\Gamma$ from a subset $Z$ of complex numbers with $\operatorname{Re}(z)>0, \forall z \in Z$ is defined by

$$
\Gamma(z)=\int_{0}^{\infty} t^{z-1} e^{-t} d t
$$

We will proceed to introduce those formulas which undoubtedly generates the $n^{\text {th }}$ term of the much selected peculiar types of real sequences. The simple proofs which are based on properties of arithmetic and geometric progressions are included and an example given in each case for illustrative purpose.

\section{Results for Real Sequences}

Proposition 3.1 Let $\left\{a_{n}\right\}_{n \geq 1}$ be a real sequence. Suppose, $\exists$ a real number $d_{2}$ satisfying

$$
d_{2}=a_{n+2}-2 a_{n+1}+a_{n}, \forall n \in \mathbb{N}
$$

Then with $d_{1}=a_{2}-a_{1}$, the $n^{\text {th }}$ term of the sequence is given by

$$
a_{n}=a_{1}+\frac{n-1}{2}\left[2 d_{1}+(n-2) d_{2}\right]
$$

Proof:

Let $\left\{a_{n}\right\}_{n \in \mathbb{N}}$ be a real sequence. Given that, $d_{2}=a_{n+2}-2 a_{n+1}+a_{n}, \forall n \in \mathbb{N}$, then

$$
d_{2}=\left(a_{n+2}-a_{n+1}\right)-\left(a_{n+1}-a_{n}\right), \forall n \in \mathbb{N},
$$

Thus, $\left\{a_{n+1}-a_{n}\right\}_{n \in \mathbb{N}}$ is an Arithmetic sequence with common difference $d_{2}$. Given that $d_{1}=a_{2}-a_{1}$, we have

$$
a_{n+1}-a_{n}=d_{1}+(n-1) d_{2}
$$

Similarly, we have

$$
\begin{aligned}
& \sum_{i=1}^{n}\left(a_{i+1}-a_{i}\right)=\frac{n}{2}\left\{2 d_{1}+(n-1) d_{2}\right\} \\
& \Rightarrow a_{n+1}-a_{1}=\sum_{i=1}^{n}\left(a_{i+1}-a_{i}\right)=\frac{n}{2}\left\{2 d_{1}+(n-1) d_{2}\right\}
\end{aligned}
$$

Solving equations (3.1.1) and (3.1.2), we can readily get the $n^{\text {th }}$ term of the sequence which is given in following equation: 


$$
a_{n}=a_{1}+\frac{n-1}{2}\left[2 d_{1}+(n-2) d_{2}\right]
$$

Hence the proof is complete.

Example 1: Obtain the $n^{\text {th }}$ term of the sequence $-4,9,-18,31, \ldots$

Solution: Let $a_{n}$ denote the $n^{\text {th }}$ term of the sequence. The alternate sign in the terms of the sequence suggests that the $n^{\text {th }}$ term will be of the form:

$$
a_{n}=(-1)^{n} b_{n} \text {, where } b_{n}=\left|a_{n}\right|, \forall n \in \mathbb{N} .
$$

For $n=1 ; \quad b_{n+2}-2 b_{n+1}+b_{n}=18-18+4=4$,

For $n=2 ; \quad b_{n+2}-2 b_{n+1}+b_{n}=31-36+9=4$,

We wish to obtain an $n$th term such that, $b_{n+2}-2 b_{n+1}+b_{n}=4, \forall n \in \mathbb{N}$. Thus,

$$
\begin{aligned}
& b_{n}=b_{1}+\frac{n-1}{2}\left[2 d_{1}+(n-2) d_{2}\right] \\
& =4+\frac{n-1}{2}[2(5)+(n-2) 4]=2 n^{2}-n+3
\end{aligned}
$$

Hence, the $n^{\text {th }}$ term of the sequence is given as below:

$$
a_{n}=(-1)^{n}\left(2 n^{2}-n+3\right)
$$

Proposition 3.2 Let $\left\{a_{n}\right\}_{n \in \mathbb{N}}$ be a real sequence. Suppose $\exists$ a real number $r$ different from zero satisfying

$$
r=\frac{a_{n+2}-a_{n+1}}{a_{n+1}-a_{n}}, \forall n \in \mathbb{N}
$$

Then with $d_{1}=a_{2}-a_{1}$, the $n^{\text {th }}$ term of the sequence is given by

$$
a_{n}=a_{1}+\frac{d_{1}\left(1-r^{n-1}\right)}{1-r}
$$

Proof:

Let $\left\{a_{n}\right\}_{n \in \mathbb{N}}$ be a real sequence. Given that, $r=\frac{a_{n+2}-a_{n+1}}{a_{n+1}-a_{n}}, \forall n \in \mathbb{N}$, then $\left\{a_{n+1}-a_{n}\right\}_{n \in \mathbb{N}}$ is a geometric sequence with common ratio $r$. Given that $d_{1}=a_{2}-a_{1}$, we have

$$
a_{n+1}-a_{n}=d_{1} r^{n-1}
$$

Similarly,

$$
\begin{aligned}
& \sum_{i=1}^{n}\left(a_{i+1}-a_{i}\right)=\frac{d_{1}\left(1-r^{n}\right)}{1-r} \\
& \Rightarrow a_{n+1}-a_{1}=\sum_{i=1}^{n}\left(a_{i+1}-a_{i}\right)=\frac{d_{1}\left(1-r^{n}\right)}{1-r}
\end{aligned}
$$

Solving equations (3.2.1) and (3.2.2), it is fairly easy to obtain a real sequence whose $n^{\text {th }}$ term is given as following:

$$
a_{n}=a_{1}+\frac{d_{1}\left(1-r^{n-1}\right)}{1-r}
$$

The proof is complete.
Example 2: Obtain the $n^{\text {th }}$ term of the sequence $-5, \frac{-9}{2},-3, \frac{3}{2}, \ldots$

Solution: Let $a_{n}$ denote the $n^{\text {th }}$ term of the sequence. Now,

For $n=1 ; \quad \frac{a_{n+2}-a_{n+1}}{a_{n+1}-a_{n}}=\frac{-3-\left(-\frac{9}{2}\right)}{-\frac{9}{2}-(-5)}=3$,

For $n=2 ; \quad \frac{a_{n+2}-a_{n+1}}{a_{n+1}-a_{n}}=\frac{\frac{3}{2}-(-3)}{-3-\left(-\frac{9}{2}\right)}=3$,

We wish to obtain an $n$th term such that, $\frac{a_{n+2}-a_{n+1}}{a_{n+1}-a_{n}}=3, \forall n \in \mathbb{N}$. Thus, we have the $n^{\text {th }}$ term as following;

$$
a_{n}=-5+\frac{\frac{1}{2}\left(1-3^{n-1}\right)}{1-3}=\frac{1}{4}\left(3^{n-1}-21\right)
$$

Proposition 3.3 Let $\left\{a_{n}\right\}_{n \in \mathbb{N}}$ be a real sequence. Suppose $\exists$ a real number $d$ satisfying the following conditions:

(i). $d=\frac{a_{n+2}}{a_{n+1}}-\frac{a_{n+1}}{a_{n}}, \forall n \in \mathbb{N}$,

(ii). $\frac{r_{1}}{d}>0$, where $r_{1}=\frac{a_{2}}{a_{1}}$.

Then denoting the Gamma function by $\Gamma$, the $n^{\text {th }}$ term of the sequence is given by

$$
a_{n}=\frac{a_{1} d^{n} \Gamma\left(n+\frac{r_{1}}{d}\right)}{\left(r_{1}+(n-1)\right) \Gamma\left(\frac{r_{1}}{d}\right)}
$$

Proof:

Let $\left\{a_{n}\right\}_{n \in \mathbb{N}}$ be a real sequence. Given that $d=\frac{a_{n+2}}{a_{n+1}}-\frac{a_{n+1}}{a_{n}}, \forall n \in \mathbb{N}$, then $\left\{\frac{a_{n+1}}{a_{n}}\right\}_{n \in \mathbb{N}}$ is an arithmetic sequence with common difference $d$. Given that $r_{1}=\frac{a_{2}}{a_{1}}$, we have

$$
\begin{gathered}
\frac{a_{n+1}}{a_{n}}=r_{1}+(n-1) d \\
\text { Similarly, } \prod_{i=1}^{n}\left(\frac{a_{i+1}}{a_{i}}\right)=\frac{d^{n} \Gamma\left(n+\frac{r_{1}}{d}\right)}{\Gamma\left(\frac{r_{1}}{d}\right)} \text {, provided } \frac{r_{1}}{d}>0 \\
\Rightarrow \frac{a_{n+1}}{a_{1}}=\prod_{i=1}^{n}\left(\frac{a_{i+1}}{a_{i}}\right)=\frac{d^{n} \Gamma\left(n+\frac{r_{1}}{d}\right)}{\Gamma\left(\frac{r_{1}}{d}\right)}
\end{gathered}
$$


Solving equations (3.3.1) and (3.3.2) simultaneously yields the $n^{\text {th }}$ term of the sequence as following:

$$
a_{n}=\frac{a_{1} d^{n} \Gamma\left(n+\frac{r_{1}}{d}\right)}{\left(r_{1}+(n-1) d\right) \Gamma\left(\frac{r_{1}}{d}\right)}
$$

The proof is complete.

Example 3: (i) Obtain then $n^{\text {th }}$ term of the sequence $3,1, \frac{2}{3}, \frac{2}{3}, \ldots$

(ii) Find the $9^{\text {th }}$ term of the sequence.

Solution: (i) Let $a_{n}$ denote the $n^{\text {th }}$ term of the sequence. Now,

For $n=1 ; \quad \frac{a_{n+2}}{a_{n+1}}-\frac{a_{n+1}}{a_{n}}=\frac{\left(\frac{2}{3}\right)}{1}-\frac{1}{3}=\frac{1}{3}$,

For $n=2 ; \quad \frac{a_{n+2}}{a_{n+1}}-\frac{a_{n+1}}{a_{n}}=\frac{\left(\frac{2}{3}\right)}{\left(\frac{2}{3}\right)}-\frac{\left(\frac{2}{3}\right)}{1}=\frac{1}{3}$,

We wish to obtain an $n$th term such that, $\frac{a_{n+2}}{a_{n+1}}-\frac{a_{n+1}}{a_{n}}=\frac{1}{3}, \forall n \in \mathbb{N}$. Thus,

$$
a_{n}=\frac{\frac{1}{3^{n+1}} \Gamma(n+1)}{\frac{n}{3} \Gamma(1)}=3^{2-n}(n-1) !
$$

(ii) By substituting $\mathrm{n}=9$ in the $n^{\text {th }}$ term of the sequence, one can easily find the $9^{\text {th }}$ term of the sequence as given below:

$$
a_{9}=3^{2-9}(9-1) !=\frac{8 !}{3^{7}}
$$

Proposition 3.4 Let $\left\{a_{n}\right\}_{n \in \mathbb{N}}$ be a real sequence. Suppose $\exists$ a real number $r$ satisfying

$$
r=\frac{a_{n+2} a_{n}}{\left(a_{n+1}\right)^{2}}, \forall n \in \mathbb{N}
$$

If $r_{1}=\frac{a_{2}}{a_{1}}>0$, then the $n^{\text {th }}$ term of the sequence is given by

$$
a_{n}=a_{1}\left(r_{1}^{2} r^{n-2}\right)^{\frac{n-1}{2}}
$$

Proof:

Let $\left\{a_{n}\right\}_{n \in \mathbb{N}}$ be a real sequence. Given that $r=\frac{a_{n+2} a_{n}}{\left(a_{n+1}\right)^{2}}, \forall n \in \mathbb{N}$, then

$$
r=\frac{a_{n+2}}{a_{n+1}} \div \frac{a_{n+1}}{a_{n}}, \forall n \in \mathbb{N}
$$

Thus, $\left\{\frac{a_{n+1}}{a_{n}}\right\}_{n \in \mathbb{N}}$ is a geometric sequence with common ratio $r$. Given that $r_{1}=\frac{a_{2}}{a_{1}}$, we have

$$
\frac{a_{n+1}}{a_{n}}=r_{1} r^{n-1}
$$

Similarly, $\prod_{i=1}^{n} \frac{a_{i+1}}{a_{i}}=r_{1}^{n+1} r^{\frac{n(n+1)}{2}}$, provided $r>0$

$$
\Rightarrow \frac{a_{n+1}}{a_{1}}=\prod_{i=1}^{n} \frac{a_{i+1}}{a_{i}}=r_{1}^{n+1} r^{\frac{n(n+1)}{2}}
$$

Solving equations (3.4.1) and (3.4.2) simultaneously yields the $n^{\text {th }}$ term of the sequence as given below:

$$
a_{n}=a_{1}\left(r_{1}^{2} r^{n-2}\right)^{\frac{n-1}{2}}
$$

The proof is complete.

Example 4: (i) Obtain the $n^{\text {th }}$ term of the sequence $6,2,1, \frac{3}{4}, \ldots$

(ii) Find the $11^{\text {th }}$ term of the sequence.

Solution: (i) Let $a_{n}$ denote the $n^{\text {th }}$ term of the sequence. Now, we have

For $n=1 ; \quad \frac{a_{n+2} a_{n}}{\left(a_{n+1}\right)^{2}}=\frac{1(6)}{2^{2}}=\frac{3}{2}$,

For $n=2 ; \quad \frac{a_{n+2} a_{n}}{\left(a_{n+1}\right)^{2}}=\frac{\left(\frac{3}{4}\right) \times 2}{1^{2}}=\frac{3}{2}$,

We wish to obtain an $n$th term such that, $\frac{a_{n+2} a_{n}}{\left(a_{n+1}\right)^{2}}=\frac{3}{2}, \forall n \in \mathbb{N}$. Thus,

$$
a_{n}=6\left(\left(\frac{1}{3}\right)^{2}\left(\frac{3}{2}\right)^{n-2}\right)^{\frac{n-1}{2}}=\left(2^{-n^{2}+3 n} 3^{n^{2}-5 n+6}\right)^{\frac{1}{2}}
$$

(ii) The $11^{\text {th }}$ term of the sequence is

$$
a_{11}=\left(2^{-11^{2}+3(11)} 3^{11^{2}-5(11)+6}\right)^{\frac{1}{2}}=\frac{3^{36}}{2^{44}}
$$

\section{Discussions and Conclusions}

Here, the $n^{\text {th }}$ term of certain class of real sequences have been obtained successfully by using the arithmetic progression (AP) and geometric progression (GP) and gamma function. Several propositions for the the $n^{\text {th }}$ term of some real sequences are examined by sufficient number of numerical examples. The $n^{\text {th }}$ term of certain class of real sequences explored herein have some characteristics of the well-known arithmetic progression (AP) and geometric progression (GP) embedded in them. It is also remarkable that the explored results in the present work 
are entirely distinguished from the trial and error approach. In addition to this, we highly expect that these ultimate findings will go a long way in enhancing the sufferings of students in their attempts to achieve this goal of finding terms of sequences in this category. Finally, with passing remarks we suggest that by following same or its extended version of our methodology the $n^{\text {th }}$ term of some other classes of real sequences can be carried out in future research.

\section{References}

[1] Abolbashari M. and Aghaeinia H. (2003), Design and analysis of a new sequence set by using chaotic dynamic systems for spread spectrum communication applications, Communication Technology Proceedings, 2003 International Conference on Communication Technology, IEEE Conference Publications, Vol. 2, 2003, pp. 917-921, ISBN 7-5635-0686-1

[2] Benward and Saker (2003). Music: In Theory and Practice, Vol. I, p. 111-12. Seventh Edition. ISBN 978-0-07-294262-0.

[3] Caplin, William Earl (2000), Fundamental Progressions of Harmony. Classical Form: A Theory of Formal Functions for the Instrumental Music of Haydn, Mozart and Beethoven. New York: Oxford UP, 2000. Pp. 29-31. Print. ISBN 978-0-19-514399-7
[4] Dawikins and Paul, Series and Sequences, Paul's Online Math Notes/Calc II (notes).

[5] Guu-Chang Yang, A new approach to direct sequence spreadspectrum multiple access communication systems, IEEE International Symposium on Information Theory, 21 June-1 July 1994, ISBN 0-7803-2015-8

[6] Hazewinkel and Michiel (2001), Sequence, Encyclopaedia of Mathematics, Springer, ISBN 978-1-55608-010-4.

[7] Howard Anton, Irl Bivens and Stephen Davis (2004), Calculus, $8^{\text {th }}$ Edition, John Wiley \& Sons, Inc., USA, ISBN 0-471-47244-1.

[8] Jue Yu, Lu Gan, and Liping Li (2009) Design of real sequence chaotic direct sequence spread spectrum communication system with realizable occupied band width, International Conference on Communications, Circuits and Systems, IEEE Conference Publications, pp. 282-286, ISBN 978-1-4244-4886-9

[9] Kelly, Thomas Forest (2011). Early Music: A Very Short Introduction, pp. 53-54. ISBN 978-0-19-973076-6.

[10] Mark, Christopher (2006), Tippett, Sequence, and Metaphor, Tippett Studies, pp. 96. Clarke, David, ed. ISBN 0-521-02683-0.

[11] Nico M. Temme (1996), "Special Functions: An Introduction to the Classical Functions of Mathematical Physics", John Wiley \& Sons, New York, ISBN 0-471-11313-1.

[12] Sarnecki, Mark (2010), Sequences, Harmony. Mississauga, Ont.: Frederick Harris Music, 2010, pp. 116-121, ISBN 978-1-55440270-0. 